\title{
Active Phagocytosis and Diachronic Processing of Calcium Oxalate Monohydrate Crystals in an in vitro Macrophage Model
}

\author{
Atsushi Okada ${ }^{a}$ Hiromasa Aoki $^{b}$ Daichi Onozato $^{b}$ Taiki Kato $^{a}$ \\ Tadahiro Hashita $^{b}$ Hiroshi Takase $^{c}$ Teruaki Sugino $^{a}$ Rei Unno ${ }^{a}$ \\ Kazumi Taguchi $^{a}$ Shuzo Hamamoto ${ }^{a}$ Ryosuke Ando ${ }^{a}$ Kentaro Mizuno ${ }^{d}$ \\ Keiichi Tozawa ${ }^{a}$ Tamihide Matsunaga ${ }^{b}$ Kenjiro Kohri ${ }^{a}$ Takahiro Yasui ${ }^{a}$ \\ a Department of Nephro-Urology, Nagoya City University Graduate School of Medical \\ Sciences, Nagoya, Japan; bepartment of Clinical Pharmacy, Graduate School of \\ Pharmaceutical Sciences, Nagoya City University, Nagoya, Japan; ' Core Laboratory, Nagoya \\ City University Graduate School of Medical Sciences, Nagoya, Japan; ${ }^{d}$ Department of Pediatric \\ Urology, Nagoya City University Graduate School of Medical Sciences, Nagoya, Japan
}

\section{Keywords}

Calcium oxalate $\cdot$ Kidney $\cdot$ Macrophages $\cdot$ Nephrolithiasis $\cdot$ Phagocytosis

\begin{abstract}
Background: We previously discovered that renal macrophages (M $\varphi s)$ phagocytose renal calcium oxalate monohydrate (COM) crystals. This study investigated the processing of engulfed crystals using in vitro models. Methods: J774.1 mouse M $\varphi s$ were exposed to COM crystals and observed for $24 \mathrm{~h}$ using polarized light microscopy with/without cytochalasin B (CB), an inhibitor of phagocytosis, to confirm active crystal phagocytosis. LysoTracker and immunohistochemical staining using transmission electron microscopy for lysosomal-associated membrane protein 1 were used to confirm engulfed COM crystal uptake into lysosomes. Diachronic tracking of specific $M \varphi s$ was performed to capture the entire course of engulfed COM crystal processing using polarized light microscopy. Follow-up studies of fluorescent $\mathrm{COM}(\mathrm{f}-\mathrm{COM}$ ) crystals using imaging cytometry were performed in the presence and absence of nigericin to dissipate the $\mathrm{pH}$ gradient in acidic organelles. Results: Phagocytosis rates increased with COM density and were significantly lower in cells treated with $\mathrm{CB}(p<0.01)$. We observed that engulfed crystals colocalized within lysosomes of the M $\varphi s$; moreover, diachronic observation indicated that the engulfed COM crystals were subdivided during $M \varphi$ division and eliminated by the 7th day of culture. Additionally, imaging cytometry showed that the fluorescence level of $\mathrm{f}$-COM crystals in the nigericin (-) group after $48 \mathrm{~h}$ was significantly lower than that in the nigericin (+) group. Conclusions: This study confirmed active phagocytosis and lysosomal processing of engulfed COM crystals by M $\varphi s$. This discovery is expected to contribute to the development of future drugs that enhance the COM crystal phagocytic ability of $M \varphi s$.




\section{Kidney \\ Blood Pressure \\ Research}

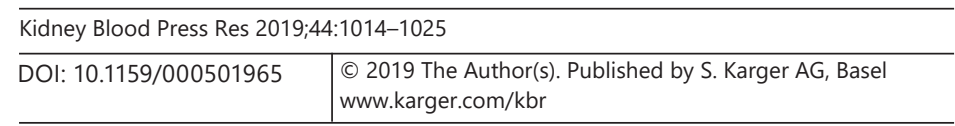

Okada et al.: Crystal Phagocytosis and Processing of M $\mathrm{Ms}$

\section{Introduction}

Clinical dissolution therapies for the cystine and uric acid components of kidney stones have been used for $>50$ years $[1,2]$; however, dissolution therapy for calcium oxalate crystals, which represent the majority of stone components, is not currently available.

The spontaneous elimination of renal intratubular calcium oxalate monohydrate (COM) crystals was previously investigated using a hyperoxaluric mouse model established by our laboratory [3]. To examine the gene-expression profiles in stone-forming mouse kidneys, we subsequently performed microarray analysis and identified characteristic changes in macrophage $(\mathrm{M} \varphi)$-related inflammation gene groups using clustering, gene ontology, and pathway analyses [4]. Additionally, ultrastructural and immunohistochemical observations of the mouse kidneys demonstrated migration and crystal phagocytosis in renal interstitial $\mathrm{M} \varphi \mathrm{s}$ [5].

Following our discovery of the "stone elimination phenomenon," several reports were published suggesting morphological disappearance of the renal crystals in vitro [6], as well as in rats and humans [7]. Therefore, this study elucidated the processing mechanisms of engulfed COM crystals using in vitro $\mathrm{M} \varphi$ models with the goal of improving treatment options for patients suffering from kidney stones.

\section{Materials and Methods}

\section{Culture of J774.1 Cells}

J774.1 mouse cultured $\mathrm{M} \varphi$ cells (Japanese Collection of Research Bioresources Cell Bank, Tokyo, Japan) were cultured in Dulbecco's modified Eagle medium (Wako Pure Chemical Industries, Ltd., Osaka, Japan) containing 10\% sterile fetal bovine serum (HanaNesco Bio Corp., Tokyo, Japan), 60 g/mL ampicillin sodium (Meiji Seika Pharma Co., Ltd., Tokyo, Japan), and $50 \mu \mathrm{g} / \mathrm{mL}$ kanamycin sulfate (Meiji Seika Pharma Co., Ltd.). Cells were split to between $0.5 \times 10^{5}$ cells $/ \mathrm{mL}$ and $1 \times 10^{5}$ cells $/ \mathrm{mL}$ every third or fourth day using 25 $\mathrm{cm}^{2}$ cell-culture flasks (Becton, Dickinson and Co., Franklin Lakes, NJ, USA) in a $5 \% \mathrm{CO}_{2}$ incubator at $37^{\circ} \mathrm{C}$.

\section{Preparation of COM Crystals}

Preparation of COM crystals was performed according to a previous report [9]. Briefly, oxalic acid sodium ( $200 \mathrm{~mm}, 0.5 \mathrm{~mL})$ and calcium chloride $(200 \mathrm{~mm}, 0.5 \mathrm{~mL})$ were mixed in a buffer containing $90 \mathrm{~mm}$ Tris- $\mathrm{HCl}$ and $10 \mathrm{~mm} \mathrm{NaCl}\left(\mathrm{pH} \mathrm{7.4)}\right.$ ) at room temperature (RT; $20^{\circ} \mathrm{C}$ ) to a final concentration of $10 \mathrm{~mm}$. The COM crystals were equilibrated for 3 days, washed 3 times with sodium- and chloride-free distilled water, saturated with calcium oxalate, and resuspended to a final concentration of $2.92 \mathrm{mg} / \mathrm{mL}$, followed by adjustment to $\mathrm{pH} 6.8$.

\section{Preparation of the Cell Suspension}

J774.1 cells were centrifuged at RT $\left(20^{\circ} \mathrm{C}\right)$ at $190 \mathrm{~g}$ for $8 \mathrm{~min}$, the supernatant was removed by decanting and discarded, and cells were collected as pellets. Following the addition of $10 \mathrm{~mL}$ culture medium, cells were centrifuged as described previously, and pellets were collected. Subsequently, $5 \mathrm{~mL}$ culture medium was added to the cell pellets, $200 \mu \mathrm{L}$ of each suspension was transferred to a $1.7 \mathrm{~mL}$ centrifuge tube (Nippon Genetics Co. Ltd., Tokyo, Japan), and cell counts and survival rates were determined. When the survival rates exceeded $90 \%$, the cell suspensions were used for subsequent examination. The cell numbers and concentrations required for each experiment were established based on the measured cell counts. 


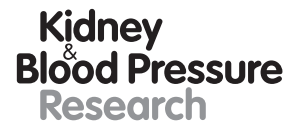

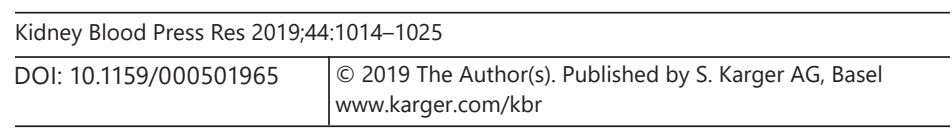

Okada et al.: Crystal Phagocytosis and Processing of M $\varphi s$

\section{Phagocytosis of COM Crystals}

J774.1 cells were prepared as cell suspensions at $5 \times 10^{5}$ cells $/ \mathrm{mL}$, placed in an 8 chamber polystyrene vessel tissue culture-treated glass slide (Becton, Dickinson and Co.) at $200 \mu \mathrm{L} /$ well, and incubated for $1 \mathrm{~h}$. The phagocytosis inhibitor cytochalasin B (CB; Sigma-Aldrich, St. Louis, MO, USA) was added to the culture medium to a final concentration of $10 \mu \mathrm{g} / \mathrm{mL}$. After a 30-min incubation, prepared COM crystals were added to the culture medium at 12.5 or 62.5 $\mu \mathrm{g} / \mathrm{cm}^{2}$. At $24 \mathrm{~h}$ following the addition of the COM crystals, the glass slides were shaken using a plate shaker for $10 \mathrm{~s}$, the supernatant was removed, and the slides were washed twice with $400 \mu \mathrm{L}$ phosphate-buffered saline (PBS[-]; pre-warmed to $37^{\circ} \mathrm{C}$; Sigma-Aldrich), from which magnesium and calcium had been removed to avoid influence on the COM crystals. After removal of the wash solution, $200 \mu \mathrm{L}$ of fresh PBS(-) containing $0.25 \%$ trypsin was added to the slide, which was subsequently placed at RT $\left(20^{\circ} \mathrm{C}\right)$ for $30 \mathrm{~min}$. Culture medium $(800 \mu \mathrm{L})$ with serum was added to the cell suspension to stop trypsin digestion, and collected cells were then centrifuged at 2,000 $\mathrm{g}$ for $5 \mathrm{~min}$, followed by removal of the supernatant.

The cell solutions were then supplemented with $200 \mu \mathrm{L}$ PBS(-) containing 4\% paraformaldehyde and fixed at RT for $30 \mathrm{~min}$. The fixed cells were centrifuged at 2,000 $\mathrm{g}$ for $5 \mathrm{~min}$, and $10 \mu \mathrm{L}$ aliquots of the fixed cell suspensions were transferred to a Burker-Turk hemocytometer (Hirschmann EM Techcolor; Hirschmann GmbH, Fluorn-winzeln, Germany). The number of intracellular COM crystals was measured using a microscope (Olympus Model CHA; Olympus, Tokyo, Japan) with a $40 \times$ objective lens. The COM crystals were counted in 2 locations on the hemocytometer, and the results were used when the total cell count exceeded 100 for each site. Based on these values, phagocytosis rates were calculated as the ratio of cells containing $>1$ COM crystal relative to the overall cell number. These studies were conducted at each COM concentration and in each group $(n=3)$.

\section{Transmission Electron Microscopy}

J774.1 cells were seeded in 6 well plates $\left(1 \times 10^{5}\right.$ cells/well) in DMEM containing $10 \%$ fetal bovine serum $+1 \%$ penicillin/streptomycin. After overnight incubation, cells were exposed to COM crystals at $62.5 \mu \mathrm{g} / \mathrm{cm}^{2}$ for $24 \mathrm{~h}$, and samples were prefixed with $2.5 \%$ glutaraldehyde in $0.1 \mathrm{M}$ phosphate buffer ( $\mathrm{pH} 7.4$ ) at $4{ }^{\circ} \mathrm{C}$. After fixation, the specimens were postfixed with $1 \%$ osmium tetroxide in $0.1 \mathrm{M}$ phosphate buffer ( $\mathrm{pH} 7.4$ ) for $45 \mathrm{~min}$ and subsequently dehydrated in a graded series of ethanol and embedded in epoxy resin.

Ultrathin sections were cut using an ULTRACUT-S (Leica, Wetzlar, Germany) with a diamond knife and stained with $2 \%$ uranyl acetate in distilled water for $15 \mathrm{~min}$, followed by staining with a lead staining solution for $5 \mathrm{~min}$. Sections were examined using a JEM-1400 plus (JEOL, Tokyo, Japan) electron microscope at $100 \mathrm{kV}$.

\section{Immunohistochemical Staining for a Lysosome Marker using Transmission Electron}

\section{Microscopy}

Following the exposure of COM crystals at $62.5 \mu \mathrm{g} / \mathrm{cm}^{2}$ on J774.1 cells for $24 \mathrm{~h}$, the cells were washed with PBS for 1 min and fixed with PBS containing 4\% paraformaldehyde for 10 min at RT $\left(20^{\circ} \mathrm{C}\right)$. After washing 3 times with PBS, the following membrane permeation treatments were sequentially performed: freeze and thaw 3 times, tris-buffered saline (TBS) containing $25 \mu \mathrm{M}$ digitonin for $5 \mathrm{~min}$, TBS containing $10 \mu \mathrm{M}$ digitonin for $5 \mathrm{~min}$, freeze and thaw 2 times, TBS containing $25 \mu \mathrm{M}$ digitonin for $10 \mathrm{~min}$, and TBS containing $10 \mu \mathrm{M}$ digitonin for 10 min. After washing 3 times with TBS, $100 \mu \mathrm{L}$ of blocking solution (TBC containing $20 \%$ of Block Ace (Dainipponseiyaku, Suita, Japan) and $0.3 \mathrm{M}$ glycine) was added for $30 \mathrm{~min}$ at RT.

The cells were then incubated overnight with polyclonal antilysosomal-associated membrane protein 1 rabbit IgG (abcam Co., Ltd., Gunma, Japan) 300 fold diluted in TBS solution containing $20 \%$ of Block Ace at $4{ }^{\circ} \mathrm{C}$. After washing 3 times with TBS, the secondary 


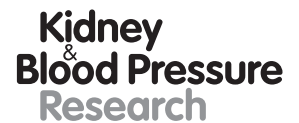

Kidney
Blood Pressure

Research \begin{tabular}{l|l}
\hline Kidney Blood Press Res 2019;44:1014-1025 \\
\hline DOI: 10.1159/000501965 & $\begin{array}{l}\text { @ 2019 The Author(s). Published by S. Karger AG, Basel } \\
\text { www.karger.com/kbr }\end{array}$ \\
\hline
\end{tabular}

Okada et al.: Crystal Phagocytosis and Processing of M $\varphi s$

antibody, anti-IgG, Rabbit, Goat-Poly, Nanogold, $\varphi 1.4 \mathrm{~nm}$ (Nanoprobes, Inc., Yaphank, NY, USA), 100 fold diluted TBS containing $20 \%$ of Block Ace were added for $2 \mathrm{~h}$ at RT $\left(20^{\circ} \mathrm{C}\right)$. After washing 5 times with TBS for $3 \mathrm{~min}$, the signals were confirmed by fluorescence microscopy.

Subsequently, the cells were prefixed with $2 \%$ glutaraldehyde in $0.1 \mathrm{M}$ phosphate buffer for $30 \mathrm{~min}$. After washing 3 times with distilled water for $3 \mathrm{~min}$, the samples were reacted with Silver Enhancement Kit HQ Silver (Nanoprobes, Inc.) for 6 min. After washing 3 times with distilled water for $3 \mathrm{~min}$, the samples were reacted with $0.05 \%$ chloroauric acid (Muto Pure Chemicals Co., Ltd., Tokyo, Japan) for 2 min. After washing 3 times with distilled water for $2 \mathrm{~min}$, the samples were postfixed with $0.1 \%$ osmium tetroxide in $0.1 \mathrm{M}$ phosphate buffer (pH 7.4) for $30 \mathrm{~min}$ and subsequently dehydrated in a graded series of ethanol and embedded in epoxy resin. Thereafter, ultrathin sectioning and observations were performed in the same manner as for transmission electron microscopy (TEM).

\section{Staining of COM Crystal-Engulfed Cells with LysoTracker}

Preparation of the J771.1 cell suspension and COM crystal exposure were performed as described above. After incubation for the stated period, cells were washed with $2 \mathrm{~mL}$ PBS(-) and warmed to $37^{\circ} \mathrm{C}$, followed by the addition of $2 \mathrm{~mL}$ culture medium containing $50 \mathrm{~nm}$ LysoTracker Red DND-99 (Invitrogen, Carlsbad, CA, USA) [8]. Following a 90-min incubation, the cells were washed with medium and then with $2 \mathrm{~mL}$ PBS(-). After aspirating the PBS and the addition of $1 \mathrm{~mL}$ fresh PBS(-), the cells were observed by light field, polarization, and fluorescence microscopy using an Eclipse TE2000-E confocal microscope (Nikon, Tokyo, Japan) with green excitation as the fluorescence filter.

\section{Diachronic Observation of Engulfed COM Crystals}

J774.1 cells were prepared in suspension at $5 \times 10^{5}$ cells $/ \mathrm{mL}$ and placed into 12 well plates at $1 \mathrm{~mL} /$ well. After a 1-h incubation, COM crystals were added to the culture medium at $12.5 \mu \mathrm{g} /$ well. After $24 \mathrm{~h}$, the 12 well plates were shaken for $10 \mathrm{~s}$, the medium was removed using an aspirator, and the plates were washed twice with $1 \mathrm{~mL}$ PBS(-) that had been warmed to $37^{\circ} \mathrm{C}$. After removal of the PBS(-), $700 \mu \mathrm{L}$ of fresh PBS(-) containing $0.25 \%$ trypsin was added, and the reaction was allowed to occur at RT $\left(20^{\circ} \mathrm{C}\right)$ for $30 \mathrm{~min}$. The cells were then separated from the plate surface by pipetting, and the resulting cell suspension was transferred to $1.7-\mathrm{mL}$ tubes containing $700 \mu \mathrm{L}$ culture medium with serum to stop the trypsin digestion. After centrifugation at 2,000 $\mathrm{g}$ for $5 \mathrm{~min}, 1 \mathrm{~mL}$ PBS(-) was added, and samples were centrifuged for $5 \mathrm{~min}$ at $2,000 \mathrm{~g}$, and the supernatant was removed. Subsequently, $500 \mu \mathrm{L}$ of culture medium containing serum was added, and cells were counted using $10 \mu \mathrm{L}$ of the sample. Cell suspensions at $2 \times 10^{3}$ cells $/ \mathrm{mL}$ were prepared and added to $35 \mathrm{~mm}$ glass-base dishes (Iwaki; Asahi Glass Co., Ltd., Tokyo, Japan) at $2 \mathrm{~mL} /$ well. Using a confocal microscope, diachronic observation of the cells was performed using a light field and polarization.

\section{Follow-Up Analysis of Fluorescent COM Crystals Phagocytozed by M $\varphi$ s Following} Inhibition of Intracellular Acidification

To capture the disappearance of COM crystals phagocytozed by J774.1 cells, we used the intracellular acidification inhibitor nigericin. COM crystals were fluorescently labeled according to the method of Chaiyarit et al. [10]. Briefly, $5 \mathrm{~mm} \mathrm{CaCl} 2 \times 2 \mathrm{H}_{2} \mathrm{O}$ was mixed with $0.5 \mathrm{mM} \mathrm{Na}_{2} \mathrm{C}_{2} \mathrm{O}_{4}$ in a buffer containing $90 \mathrm{~mm}$ Tris- $\mathrm{HCl}$ and $10 \mathrm{~mm} \mathrm{NaCl}$ (pH 7.4). The solutions were incubated at $25^{\circ} \mathrm{C}$ overnight, and COM crystals were harvested by centrifugation at $2,000 \mathrm{~g}$ for $5 \mathrm{~min}$. The supernatant was discarded, and COM crystals were resuspended in methanol. After another round of centrifugation at 2,000 $\mathrm{g}$ for $5 \mathrm{~min}$, the methanol was discarded, and the crystals were air-dried overnight at RT $\left(20^{\circ} \mathrm{C}\right)$. For staining/labeling, COM 


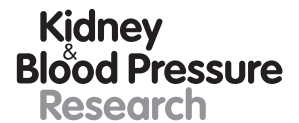

Kidney
Blood Pressure
Research

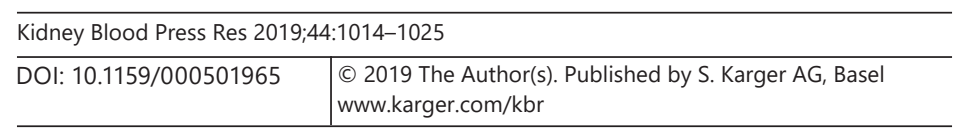

Okada et al.: Crystal Phagocytosis and Processing of M $\varphi s$

crystals were crystallized in the presence of $0.11 \mathrm{mg} / \mathrm{mL}$ AlexaFluor 488 (Thermo Fisher Scientific, Burlington, ON, Canada). The prepared fluorescent COM (f-COM) crystals were capable of identification at an excitation wavelength of $488 \mathrm{~nm}$ and an emission wavelength of $519 \mathrm{~nm}$ was prepared.

J774.1 cells were cultured to semi-confluence with DMEM + penicillin/streptomycin medium in a $10 \mathrm{~cm}$ Petri dish, followed by the addition of $62.5 \mu \mathrm{g} / \mathrm{mL} \mathrm{f}-\mathrm{COM}$ crystals and incubation for $24 \mathrm{~h}$. After washing twice with PBS, $4 \times 10^{4}$ cells/well were harvested in 60 wells of a 96 well plate coated with $0.1 \%$ gelatin and incubated at $4{ }^{\circ} \mathrm{C}$ overnight. In 30 of the wells, $2 \mu \mathrm{mol} / \mathrm{L}$ nigericin [11], a protonophore that can be used to dissipate the $\mathrm{pH}$ gradient in acidic organelles, was administered (the nigericin[+] group), with the remaining 30 wells representing the nigericin(-) group. Thereafter, live cell imaging was performed using In Cell Analyzer 6000 (GE Healthcare UK Ltd., Buckinghamshire, England), to quantify the fluorescence emission of AlexaFluor 488 during incubation under the condition of $5 \% \mathrm{CO}_{2}$ and $37^{\circ} \mathrm{C}$. The fluorescence of f-COM crystals per cells in the 60 wells was scanned simultaneously, and sequential image capture of the wells was performed every $20 \mathrm{~min}$ for $48 \mathrm{~h}(2,880 \mathrm{~min})$. After the scanning of all images, each fluorescence intensity was shown as quantified values, the average value at the 0 min time point in each group was set to 1 , and the value at each time point was expressed by a ratio.

\section{Detection of Internalization of f-COM Crystals in $M \varphi$}

This observation was performed to confirm that the fluorescence data obtained in the follow-up analysis of f-COM crystals using imaging cytometry reflected that of COM crystals intrinsic to M $\varphi$. Culture of J774.1 cells to semi-confluence and addition of f-COM crystals were performed in the same manner as for the follow-up analysis. The cells were fixed with PBS containing $4 \%$ paraformaldehyde at RT $\left(20^{\circ} \mathrm{C}\right)$ for $10 \mathrm{~min}$. After washing with PBS for $5 \mathrm{~min}$, $0.1 \%$ Triton X-100 in PBS was administered at RT $\left(20^{\circ} \mathrm{C}\right)$ for $3 \mathrm{~min}$. The cells were stained with Acti-stain TM 555 phalloidin (Cosmo Bio USA, Inc., Carlsbad, CA, USA) and 4,6-diamidino2-phenylindole, dihydrochloride (Cosmo Bio USA) at RT $\left(20^{\circ} \mathrm{C}\right.$ ) for $30 \mathrm{~min}$. After washing with PBS, the samples were scanned using the In Cell Analyzer 6000.

\section{Statistical Methods}

For the phagocytosis assay, differences were assessed using a Student $t$ test. Results were considered statistically significant at $p<0.05$. Analyses were performed using JMP version 12.2.0 (SAS Institute, Inc., Cary, NC, USA).

\section{Results}

Phagocytosis Assay of COM Crystals with or without CB

We observed that phagocytosis rates increased according to the exposed COM density $\left(19.3 \%\right.$ at COM $12.5 \mu \mathrm{g} / \mathrm{cm}^{2}$ and $39.7 \%$ at COM $\left.62.5 \mu \mathrm{g} / \mathrm{cm}^{2}\right)$ and were significantly suppressed by CB administration $\left(0.7 \%\right.$ at COM $12.5 \mu \mathrm{g} / \mathrm{cm}^{2}$ and $13.3 \%$ at COM $62.5 \mu \mathrm{g} / \mathrm{cm}^{2}$; Fig. 1).

\section{Evidence for Localization of Phagocytozed COM Crystals in Lysosomes of M $\varphi s$}

Using phase-contrast microscopy and polarized light microscopy, the majority of COM crystals after $24 \mathrm{~h}$ of exposure were observed at the same position as M $\varphi s$. Moreover, using fluorescent images, almost all LysoTracker fluorescence colocalized with the polarization of the COM crystals (Fig. 2). Additionally, TEM analysis identified COM crystals in structures surrounded by a single membrane exhibiting expression of lysosomal-associated membrane protein-1, which identified the organelles as lysosomes (Fig. 3). 
Kidney

Blood Pressure

Research

Fig. 1. Phagocytosis rates of $\mathrm{COM}$ crystals in J774.1 cells. Phagocytosis rates were calculated as the number of phagocytic cells/total cell number (\%). The J774.1 cells had a phagocytosis rate of $19.3 \pm$ $5.9 \%$ for COM at $12 \mu \mathrm{g} / \mathrm{cm}^{2}$. Administration of the phagocytosis inhibitor CB significantly decreased the rate to $0.7 \pm 1.2 \%$ ( $p=$ $0.0056)$. Additionally, the rate for $\mathrm{COM}$ at $62.5 \mu \mathrm{g} / \mathrm{cm}^{2}(39.7 \pm$ $5.51 \%)$ was significantly decreased to $13.3 \pm 3.21 \%$ through the administration of $\mathrm{CB}(p=$ 0.002). CB, cytochalasin B; COM, calcium oxalate monohydrate.

\begin{tabular}{l|l}
\hline Kidney Blood Press Res 2019;44:1014-1025 \\
\hline DOI: 10.1159/000501965 & $\begin{array}{l}\text { ○ 2019 The Author(s). Published by S. Karger AG, Basel } \\
\text { www.karger.com/kbr }\end{array}$ \\
\hline
\end{tabular}

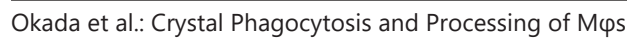

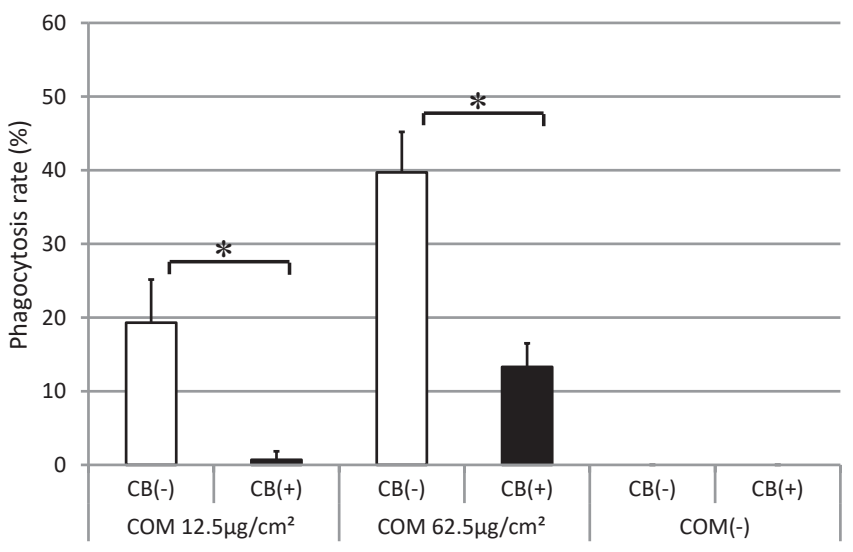

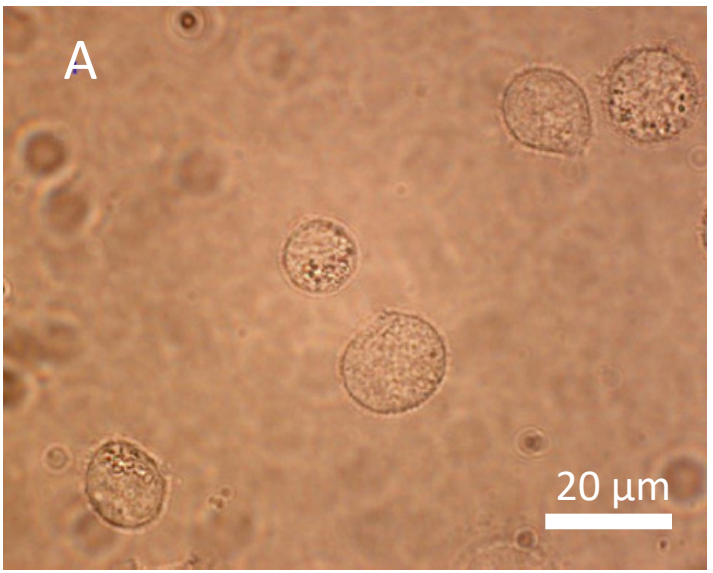
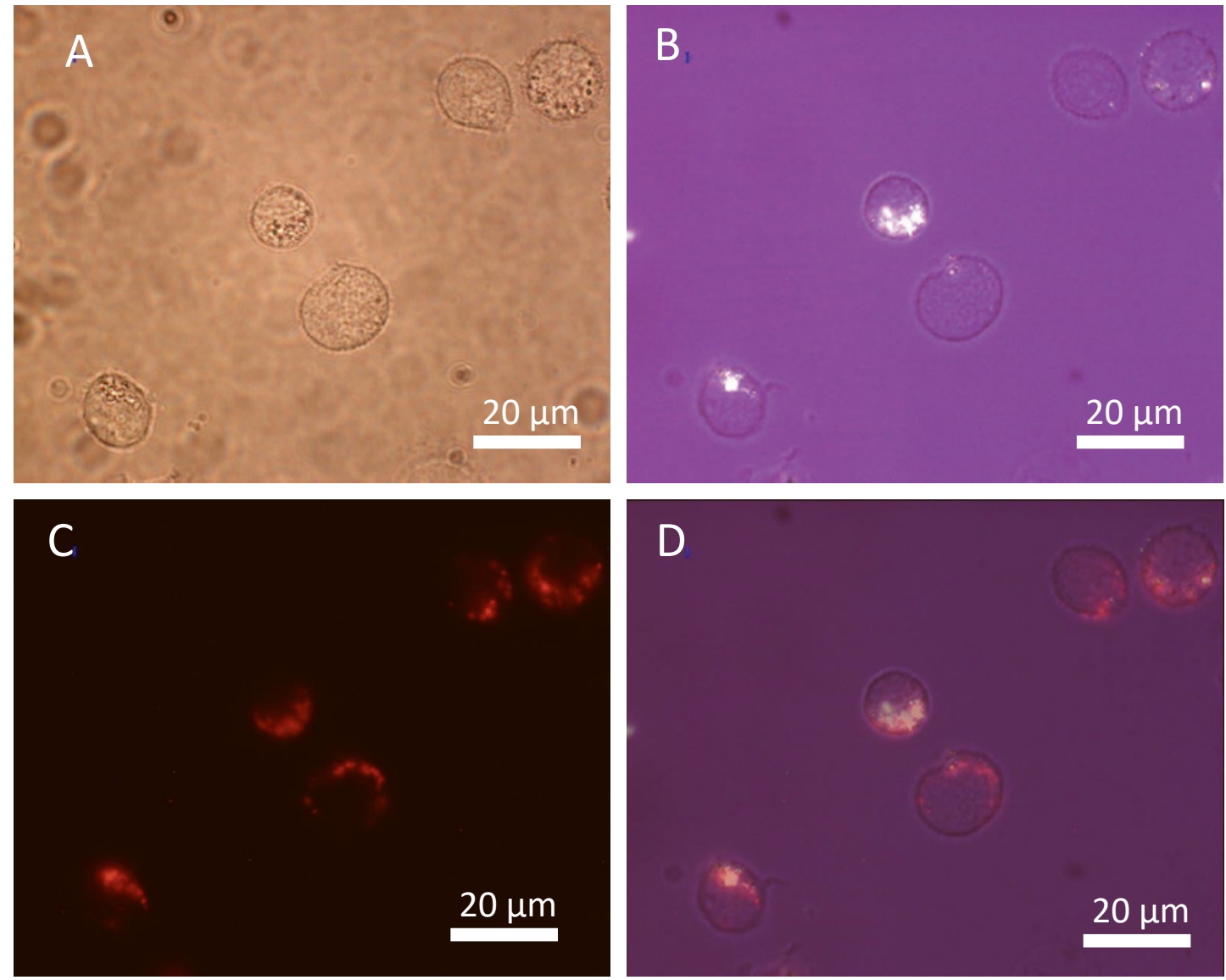

Fig. 2. LysoTracker assay for COM crystal-containing M $\varphi$ s. A Phase-contrast microscopy, (B) polarized light microscopy, and (C) fluorescent image (magnification, 400×). D Merged image of (B, C). On day 1 after exposure of J774.1 cells to COM crystals, almost all LysoTracker fluorescence colocalized with the polarization of the COM crystals. 

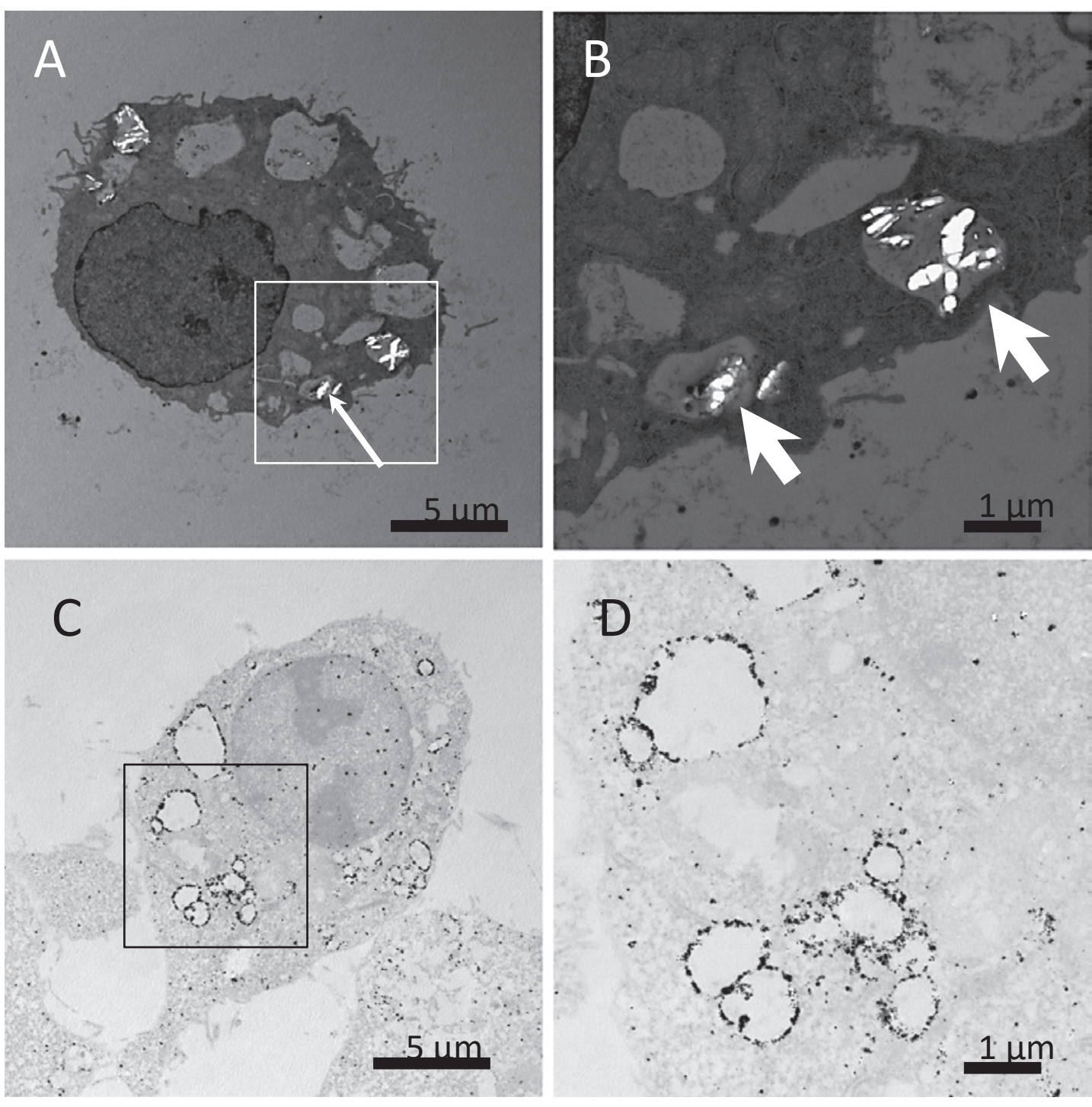

Fig. 3. TEM and immune-TEM of COM crystals phagocytozed by J774.1 cells. COM crystals were identified in structures surrounded by a single membrane. A Magnification, 1,500× (scale bar: $5.0 \mu \mathrm{m}$ ). B Magnification, 5,000× (scale bar: $1.0 \mu \mathrm{m}$ ). The arrows show COM crystals in single membrane organelles. C, D Showed immuneTEM images. C Magnification, 1,500× (scale bar: $5.0 \mu \mathrm{m}$ ). D Magnification, 5,000× (scale bar: $1.0 \mu \mathrm{m}$ ). Silver-enhanced gold particles indicating anti-LAMP-1 antibody are localized on the single membrane of lysosomes.

\section{Diachronic Observation of Engulfed COM Crystals}

Using polarized light microscopy, diachronic observation of the specific crystals revealed that many phagocytozed crystals were fragmented by the division of $M \varphi s$ and were not visible on day 6 (Fig. 4a-c), although some intracellular crystals were not divided or diminished (Fig. 4c).

Follow-Up Analysis off-COM Crystals Phagocytozed by M $\varphi$ s Following Inhibition of Intracellular Acidification

J774.1 cell incorporation of scattered peripheral crystals resulted in increased levels of f-COM fluorescence, resulting in equivalent levels in cells of both nigericin (+) and nigericin $(-)$ groups up to $8 \mathrm{~h}$ after treatment. Thereafter, the level of f-COM fluorescence gradually 


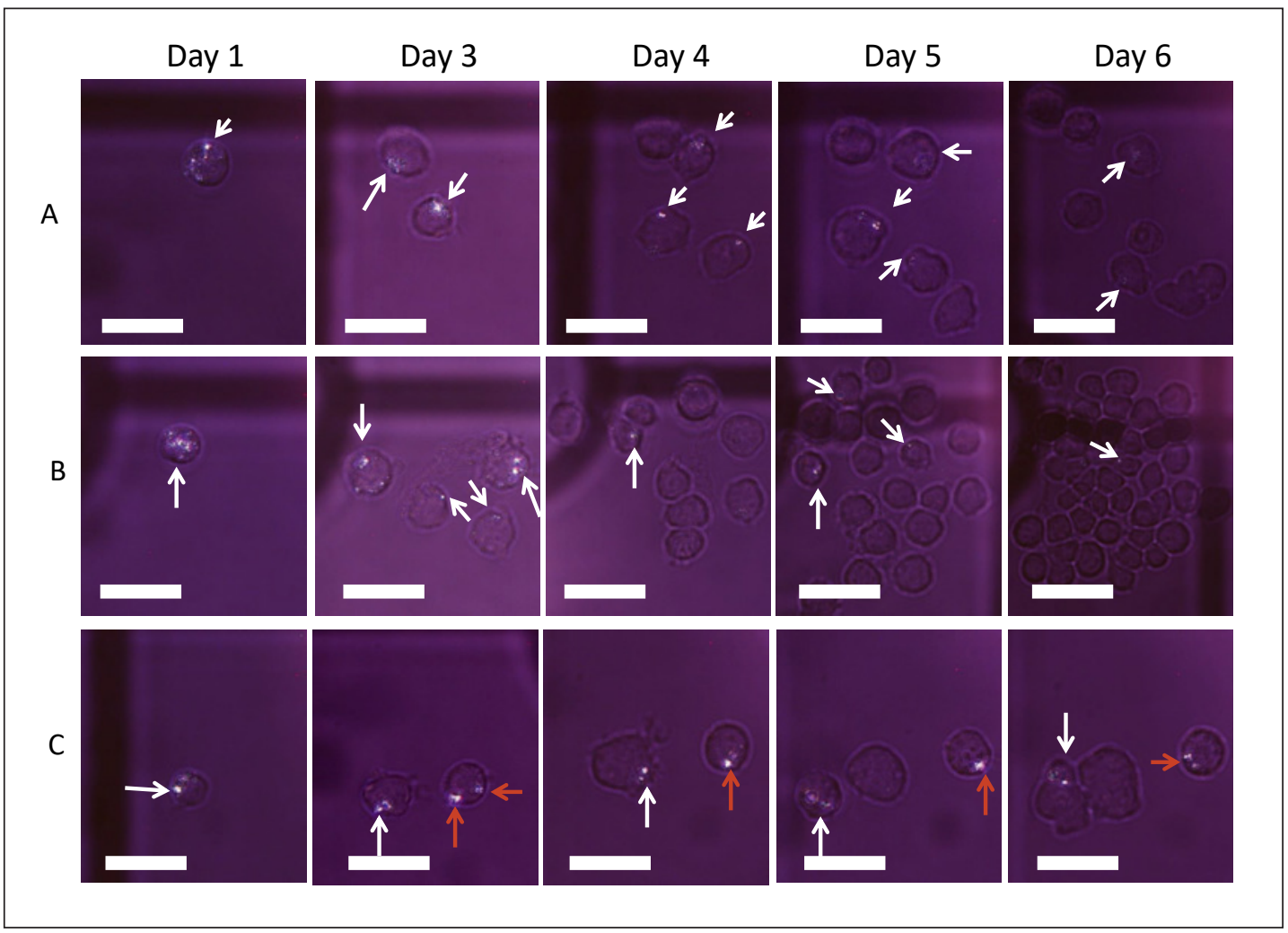

Fig. 4. Diachronic observation of engulfed COM crystals. J774.1 cells disseminated in a 35-mm glass-base dish were observed using the polarized mode of the confocal microscope. Specific cells containing COM crystals were observed at $1,3,4,5$, and 6 days after dissemination. White arrows show intracellular COM crystals that had distributed with cell division (A-C). Red arrows show intracellular COM crystals that did not separate or were diminished (C). (Scale bar: $100 \mu \mathrm{m}$ ).

decreased; however, after $48 \mathrm{~h}$, the nigericin (+) group returned to levels observed at the beginning of the experiment. Conversely, fluorescence in the nigericin(-) group significantly decreased to approximately $35 \%$ of the initial fluorescence levels after $48 \mathrm{~h}$ (Fig. 5; online suppl. Data 1; for all online suppl. material, see www.karger.com/doi/10.1159/000501965).

To confirm the internalization of $\mathrm{f}-\mathrm{COM}$ in $\mathrm{M} \varphi s$, multiple fluorescently stained $\mathrm{M} \varphi s$ were observed using an In Cell Analyzer 6000. The results revealed that f-COM crystals were present at the same site as the cytoplasm (stained red by phalloidin) and the M $\varphi s$ (stained blue by 4,6-diamidino-2-phenylindole, dihydrochloride; Fig. 6).

\section{Discussion}

Our previous studies using a hyperoxaluric nephrolithiasis model revealed the possibility of crystal processing by M $\varphi s$ [3-5]. Morphologically, interstitial M $\varphi s$ of the kidney were found to surround calcium oxalate crystals in hyperoxaluric rat models [12]. These crystalengulfing M $\varphi$ s produced the cytokine interleukin-6 [13]. Additionally, Kusmartsev et al. [14] reported calcium oxalate stone fragment and crystal phagocytosis by human M $\varphi$ s. Furthermore, we recently elucidated the gene-expression profiles of human Randall's plaque tissues, with subsequent analysis of gene networks demonstrating that these plaque tissues were associated with upregulated genes, including a relationship between $\mathrm{M} \varphi$ activation and 


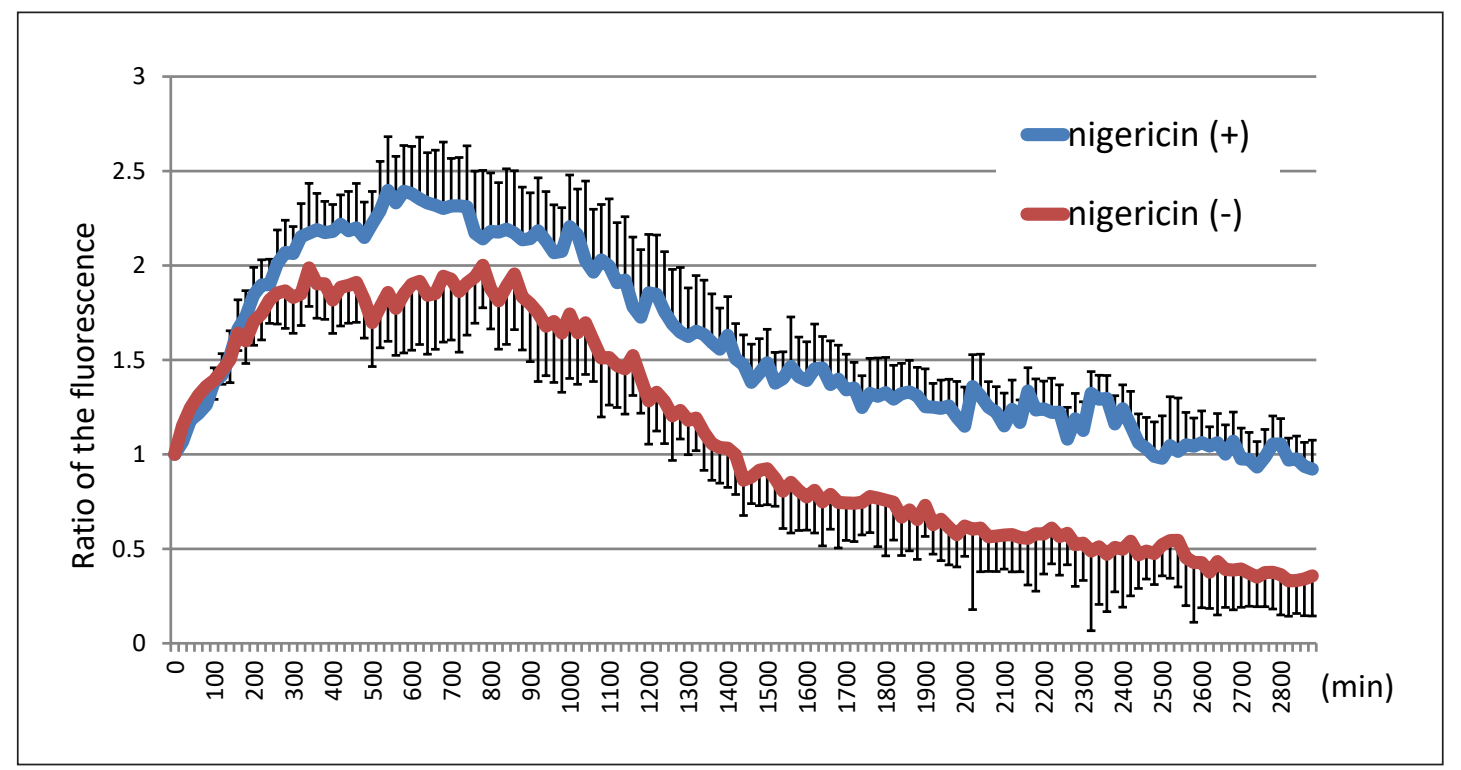

Fig. 5. Follow-up analysis of f-COM crystals phagocytozed by $\mathrm{M} \varphi$ s following inhibition by intracellular acidification. The vertical scale represents the ratio of the fluorescence of AlexaFluor 488 bound to the calcium oxalate crystals existing in the cell in the 2 -dimensional observation. The average value at the 0 min time point in each group was set to 1 , and the value at each time was expressed by a ratio. A significant difference $(p<0.05)$ according to a $t$ test was observed between groups from 300 to 2,880 min.

Fig. 6. Internalization of $\mathrm{f}-\mathrm{COM}$ in $\mathrm{M} \varphi \mathrm{s}$ using multiple fluorescently stained $\mathrm{M} \varphi$ s. The fluorescence-stained COM crystals of AlexaFluor 488 are shown as green, phalloidin-stained $\mathrm{M} \varphi \mathrm{s}$ cytoplasm as red, and DAPI-stained $\mathrm{M} \varphi$ nuclei as blue. The f-COM crystals are present at the same site as the cytoplasm (stained red by phalloidin) and the M $\varphi s$ (stained blue by DAPI). Right: magnified image of boxed area.
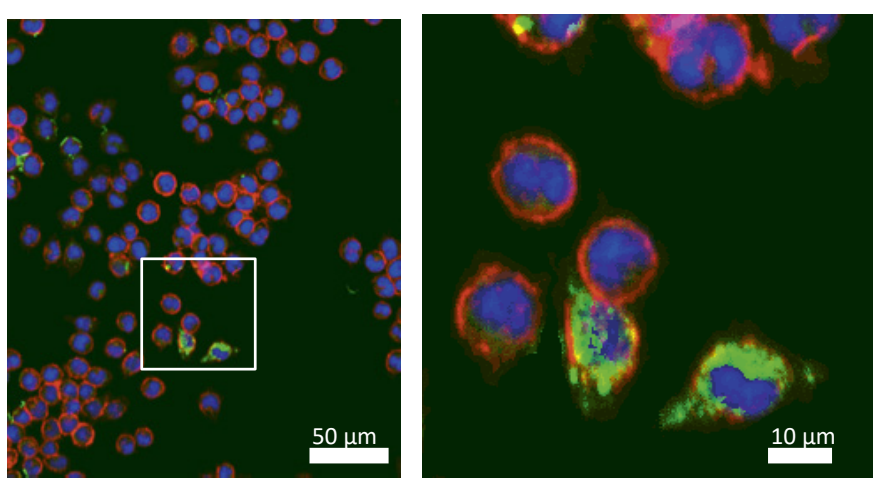

proinflammatory cytokines [15]. Based on these results, it was suggested that calcium oxalate crystal phagocytosis was likely to occur within kidney tissue.

Conversely, M $\varphi s$ have been classically recognized as having a role in kidney stone formation. In in vitro studies by Umekawa et al. $[16,17]$, the exposure of renal tubule cells to calcium oxalate crystals promoted the production of monocyte chemoattractant protein-1, suggesting that inflammation is mediated by $M \varphi s$. The similarities between the mechanisms involved in the formation of atherosclerosis and kidney stones also suggest a common inflammatory etiology. For example, susceptible ages include males of middle and old age and postmenopausal female; another cause is a westernized diet. Components include calcium and osteopontin, and both are induced by cytokines and M $\varphi$ s. The grounds for such theories are based on results from our previous studies, including the correlation between aortic calcification index and the prevalence of kidney stones [18], the positive association between kidney stone formation and conventional risk factors of coronary heart disease (as deter- 
Kidney
Blood Pressure

Research

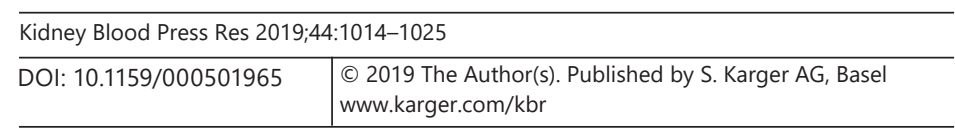

Okada et al.: Crystal Phagocytosis and Processing of $M \varphi s$

mined by a large-scale cohort study) [19], an in vitro study using cocultured $M \varphi s$ with renal epithelial cells and adipocytes [9], and an in vivo study using metabolic syndrome model animals [20]. We were also inspired by a study on the regression of atherosclerotic plaques [21], and ultimately considered that $\mathrm{M} \varphi \mathrm{s}$ might serve as a therapeutic target, potentially leading to the dissolution of kidney stones. Notably, the early phase of atherosclerotic plaque regression showed a loss of foam cells from the lesions and a concomitant increase in nonfoam cell (healthy) M $\varphi s$ surrounding areas of necrosis, in which removal of the material by an influx of functioning, healthy phagocytes was observed to occur [22]. In addition, we recently reported that kidney stone formers had more renal parenchymal crystals than nonstone formers, particularly in the papilla region, and the relationship between the crystal and $M \varphi$ number [23]. The differences in the crystal processing ability of $M \varphi s$ between healthy and stone-forming patients should be further studied.

In subsequent studies, we focused our attention on the polarity of $\mathrm{M} \varphi \mathrm{s}$ as a potential explanation for the possibility that they might function during both the promotion and suppression of kidney stone formation. Using anti-inflammatory-type $\mathrm{M} \varphi$ (M2)-deficient mice, we demonstrated that M2 cells could suppress calcium oxalate nephrolithiasis [24]. Furthermore, using an obese mouse model of metabolic syndrome, inflammatory-type (M1) M $\varphi$ s were shown to facilitate renal crystal deposition [25]. Additionally, we investigated the role of M1/M2 cells in crystal development by transfusion and M1/M2 M $\varphi$ induction, concluding that renal crystal development was facilitated by M1 M $\varphi$ s but suppressed by M2 $\mathrm{M} \varphi s$ [26]. Based on these results, we proposed the hypothesis that humans are able to remove renal crystals via M2 M $\varphi$ s, which naturally protect the kidney from clinical stone formation. In contrast, $\mathrm{M} 1 \mathrm{M} \varphi \mathrm{s}$ activated by inflammatory environments, such as that occurring during metabolic syndrome, promote kidney stone formation. Moreover, we recently performed multiplex analysis of human urine and showed that patients with calculi exhibited lower levels of IL-4, an inducer of M2-M $\varphi$, than those of healthy individuals [27].

We also reported the phenomenon of crystal phagocytosis by M $\varphi s$ [24, 26], although the detailed mechanism of crystal processing is not yet understood. In the present study, we investigated several issues that could contribute to the development of pharmaceutical strategies for crystal processing. Such issues included whether the process of crystal phagocytosis is active or passive, whether the utilized cultured cells exhibit phagocytosis, what conditions would expose engulfed crystals to $\mathrm{M} \varphi \mathrm{s}$, and whether the engulfed crystals could eventually be dissolved.

To examine these issues, we utilized cytochalasins, which bind to actin filaments and inhibit polymerization and elongation. In particular, cytochalasins B inhibit phagocytosis in neutrophils and $\mathrm{M} \varphi \mathrm{s}$. Therefore, pharmacological inhibition by cytochalasin leads to material transport within $\mathrm{M} \varphi \mathrm{s}$ instead of phagosome formation. Accordingly, the results of the phagocytosis assay with cytochalasins (Fig. 1) indicated that COM crystal transport within M $\varphi s$ is dependent upon active phagocytosis.

Generally, the materials transported within $\mathrm{M} \varphi s$ are confined to phagosomes; subsequently, phagosomes fuse with lysosomes that contain hydrolases for digestion of the internal materials [28]. We monitored engulfed COM crystals by polarized light microscopy, fluorescence microscopy, and TEM and detected their cellular position using LysoTracker (Fig. 3). Morphologically, the crystals were shown to exist within lysosomes of M $\varphi$ s (Fig. 4) and expected to be exposed to the acidified lysosomal environment. The results further indicated that COM crystals could be transported within M $\varphi$ s and were subsequently surrounded by phagosomes that fused with lysosomes. Calcium oxalate stones are likely generated under acidic urinary conditions ( $\mathrm{pH}$ 5.0-6.0) and cannot be dissolved physiologically by urine. However, some studies demonstrated that calcium oxalate could be dissolved in environments of pH 2-4 [29] and that intra-phagolysosomal conditions are likely suitable for dissolution. 


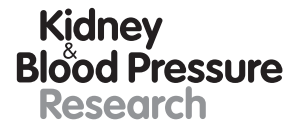

Kidney
Blood Pressure
Research

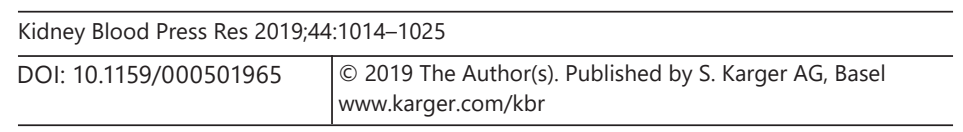

Okada et al.: Crystal Phagocytosis and Processing of M $\varphi s$

The possibility of crystal phagocytosis has been reported by several groups [7, 12, 14], as well as by our group [3-5, 24, 26]; however, actual elimination of engulfed COM crystals had not been previously detected. In the present study, we presented the first evidence of diachronic elimination of engulfed COM crystals in viable $\mathrm{M} \varphi s$ using glass-base dishes and reductions in the fluorescence intensity of $\mathrm{f}-\mathrm{COM}$ crystals in $\mathrm{M} \varphi$ s by imaging cytometry. Specifically, the f-COM study showed significant inhibition based on decreased fluorescence levels following administration of nigericin. This phenomenon is consistent with renal crystal elimination associated with interstitial $\mathrm{M} \varphi$ migration that occurs in the hyperoxaluric mouse model [5], as previously reported by our group.

There are several limitations to this study. Cultured J774.1 $\mathrm{M} \varphi$ cells were considered suitable for phagocytosis studies, as reported previously [8], although related gene expression has not been evaluated. Based on the results of this study, we considered that such expression analyses, including examining the expression of factors, such as stone matrix protein, cytokines/chemokines, and oxidative stress markers, would be essential to confirm and expand our findings. Cytochalasin is used as a phagocytosis inhibitor, but owing to its effect on actin polymerization, other potential reasons exist for lack of uptake. This includes the ability to reduce cell motility and chemotaxis [30]. In order to verify whether cell motility in the culture vessel on the glass slide or cell interaction with the crystal was significantly inhibited, the single-cell trend needed to be captured by live imaging; however, we were unable to accomplish this. Although it is unknown whether this process is active or passive, the results clearly showed that COM crystals are phagocytozed by human and animal M $\varphi s$ [14].

Overall, this study provided the first confirmation of active phagocytosis and lysosomal processing of engulfed COM crystals by M $\varphi$ s. These findings might be useful for the development of therapies for the dissolution of calcium oxalate stones.

\section{Acknowledgments}

We would like to thank Ms. Naomi Kasuga, Ms. Momoko Noda, and Ms. Ikuko Ando for their assistance with the experimental studies. This study was supported in part by Grants-in-Aid for Scientific Research from the Japan Society for the Promotion of Science (Nos. 16K15692, 16K11022, 16K20153, 16K11053, 16K11054, 16K11055, 15K20104, and 15K10626), the 1st Research Grant from the Japanese Society on Urolithiasis Research, the 8th Young Researcher Promotion Grant of the Japanese Urological Association, a Research Grant from the Mitsui Life Social Welfare Foundation and Aichi Health Promotion Foundation, a Medical Research Grant from the Takeda Science Foundation, the Medical Research Encouragement Prize from The Japan Medical Association, the 27th Research Grant from Japan Urological Association, and a Research Grant from Suzuki Urological Medicine Foundation.

\section{Disclosure Statement}

The authors have no conflicts of interest to declare.

\section{References}

Crawhall JC, Scowen EF, Watts RW. Effect of penicillamine on cystinuria. BMJ. 1963 Mar;1(5330):588-90. Gutman AB, Yü TF. Uric acid nephrolithiasis. Am J Med. 1968 Nov;45(5):756-79.

3 Okada A, Nomura S, Higashibata Y, Hirose M, Gao B, Yoshimura M, et al. Successful formation of calcium oxalate crystal deposition in mouse kidney by intraabdominal glyoxylate injection. Urol Res. 2007 Apr;35(2):89-99. 
Okada et al.: Crystal Phagocytosis and Processing of M $\varphi s$

4 Okada A, Yasui T, Hamamoto S, Hirose M, Kubota Y, Itoh Y, et al. Genome-wide analysis of genes related to kidney stone formation and elimination in the calcium oxalate nephrolithiasis model mouse: detection of stone-preventive factors and involvement of macrophage activity. J Bone Miner Res. 2009 May;24(5):908-24.

5 Okada A, Yasui T, Fujii Y, Niimi K, Hamamoto S, Hirose M, et al. Renal macrophage migration and crystal phagocytosis via inflammatory-related gene expression during kidney stone formation and elimination in mice: detection by association analysis of stone-related gene expression and microstructural observation. J Bone Miner Res. 2010 Dec;25(12):2701-11.

6 Grover PK, Thurgood LA, Fleming DE, van Bronswijk W, Wang T, Ryall RL. Intracrystalline urinary proteins facilitate degradation and dissolution of calcium oxalate crystals in cultured renal cells. Am J Physiol Renal Physiol. 2008 Feb;294(2):F355-61.

7 Vervaet BA, Verhulst A, Dauwe SE, De Broe ME, D’Haese PC. An active renal crystal clearance mechanism in rat and man. Kidney Int. 2009 Jan;75(1):41-51.

8 Beletskii A, Cooper M, Sriraman P, Chiriac C, Zhao L, Abbot S, et al. High-throughput phagocytosis assay utilizing a pH-sensitive fluorescent dye. Biotechniques. 2005 Dec;39(6):894-7.

9 Zuo L, Tozawa K, Okada A, Yasui T, Taguchi K, Ito Y, et al. A paracrine mechanism involving renal tubular cells, adipocytes and macrophages promotes kidney stone formation in a simulated metabolic syndrome environment. J Urol. 2014 Jun;191(6):1906-12.

10 Chaiyarit S, Mungdee S, Thongboonkerd V. Non-radioactive labeling of calcium oxalate crystals for investigations of crystal-cell interaction and internalization. Anal Methods. 2010;2(10):1536-41.

11 Douvdevani A, Rapoport J, Konforty A, Yulzari R, Moran A, Chaimovitz C. Intracellular acidification mediates the inhibitory effect of peritoneal dialysate on peritoneal macrophages. J Am Soc Nephrol. 1995 Aug;6(2): 207-13.

12 de Water R, Noordermeer C, Houtsmuller AB, Nigg AL, Stijnen T, Schröder FH, et al. Role of macrophages in nephrolithiasis in rats: an analysis of the renal interstitium. Am J Kidney Dis. 2000 Sep;36(3):615-25.

13 de Water R, Leenen PJ, Noordermeer C, Nigg AL, Houtsmuller AB, Kok DJ, et al. Cytokine production induced by binding and processing of calcium oxalate crystals in cultured macrophages. Am J Kidney Dis. 2001 Aug; 38(2):331-8.

14 Kusmartsev S, Dominguez-Gutierrez PR, Canales BK, Bird VG, Vieweg J, Khan SR. Calcium oxalate stone fragment and crystal phagocytosis by human macrophages. J Urol. 2016 Apr;195(4 Pt 1):1143-51.

15 Taguchi K, Hamamoto S, Okada A, Unno R, Kamisawa H, Naiki T, et al. Genome-wide gene expression profiling of Randall's plaques in calcium oxalate stone formers. J Am Soc Nephrol. 2017 Jan;28(1):333-47.

16 Umekawa T, Chegini N, Khan SR. Oxalate ions and calcium oxalate crystals stimulate MCP-1 expression by renal epithelial cells. Kidney Int. 2002 Jan;61(1):105-12.

17 Umekawa T, Chegini N, Khan SR. Increased expression of monocyte chemoattractant protein-1 (MCP-1) by renal epithelial cells in culture on exposure to calcium oxalate, phosphate and uric acid crystals. Nephrol Dial Transplant. 2003 Apr;18(4):664-9.

18 Yasui T, Itoh Y, Bing G, Okada A, Tozawa K, Kohri K. Aortic calcification in urolithiasis patients. Scand J Urol Nephrol. 2007;41(5):419-21.

19 Ando R, Nagaya T, Suzuki S, Takahashi H, Kawai M, Okada A, et al. Kidney stone formation is positively associated with conventional risk factors for coronary heart disease in Japanese men. J Urol. 2013 Apr;189(4): 1340-6.

20 Fujii Y, Okada A, Yasui T, Niimi K, Hamamoto S, Hirose M, et al. Effect of adiponectin on kidney crystal formation in metabolic syndrome model mice via inhibition of inflammation and apoptosis. PLoS One. 2013 Apr; 8(4):e61343.

21 Williams KJ, Feig JE, Fisher EA. Rapid regression of atherosclerosis: insights from the clinical and experimental literature. Nat Clin Pract Cardiovasc Med. 2008 Feb;5(2):91-102.

22 Daoud AS, Jarmolych J, Augustyn JM, Fritz KE. Sequential morphologic studies of regression of advanced atherosclerosis. Arch Pathol Lab Med. 1981 May;105(5):233-9.

23 Okada A, Hamamoto S, Taguchi K, Unno R, Sugino T, Ando R, et al. Kidney stone formers have more renal parenchymal crystals than non-stone formers, particularly in the papilla region. BMC Urol. 2018 Mar;18(1):19.

24 Taguchi K, Okada A, Kitamura H, Yasui T, Naiki T, Hamamoto S, et al. Colony-stimulating factor-1 signaling suppresses renal crystal formation. J Am Soc Nephrol. 2014 Aug;25(8):1680-97.

25 Taguchi K, Okada A, Hamamoto S, Iwatsuki S, Naiki T, Ando R, et al. Proinflammatory and metabolic changes facilitate renal crystal deposition in an obese mouse model of metabolic syndrome. J Urol. 2015 Dec;194(6): 1787-96.

26 Taguchi K, Okada A, Hamamoto S, Unno R, Moritoki Y, Ando R, et al. M1/M2-macrophage phenotypes regulate renal calcium oxalate crystal development. Sci Rep. 2016 Oct;6(1):35167.

27 Okada A, Ando R, Taguchi K, Hamamoto S, Unno R, Sugino T, et al. Identification of new urinary risk markers for urinary stones using a logistic model and multinomial logit model. Clin Exp Nephrol. 2019 May;23(5): 710-6.

28 Kapellos TS, Taylor L, Lee H, Cowley SA, James WS, Iqbal AJ, et al. A novel real time imaging platform to quantify macrophage phagocytosis. Biochem Pharmacol. 2016 Sep;116:107-19.

29 Cezar TM. Calcium oxalate: a surface treatment for limestone. J Conserv Mus Stud. 1998;4:6-10.

30 Iqbal AJ, Regan-Komito D, Christou I, White GE, McNeill E, Kenyon A, et al. A real time chemotaxis assay unveils unique migratory profiles amongst different primary murine macrophages. PLoS One. 2013;8(3):e58744. 